\title{
Editorial
}

\section{Advanced Stochastic Control Systems with Engineering Applications}

\author{
Ming Liu, ${ }^{1}$ Peng Shi, ${ }^{2}$ Hamid Reza Karimi, ${ }^{3}$ Shen Yin, ${ }^{4}$ and Xiaojie Su \\ ${ }^{1}$ School of Astronautics, Harbin Institute of Technology, Harbin, Heilongjiang, China \\ ${ }^{2}$ School of Electrical and Electronic Engineering, The University of Adelaide, SA 5005, Australia \\ ${ }^{3}$ Department of Engineering, Faculty of Engineering and Science, University of Agder, 4898 Grimstad, Norway \\ ${ }^{4}$ Institute of Automation and Complex Systems, University of Duisburg-Essen, Duisburg, Germany \\ ${ }^{5}$ College of Automation, Chongqing University, Chongqing 400044, China
}

Correspondence should be addressed to Ming Liu; mingliu23@hit.edu.cn

Received 26 May 2014; Accepted 26 May 2014; Published 12 June 2014

Copyright (C) 2014 Ming Liu et al. This is an open access article distributed under the Creative Commons Attribution License, which permits unrestricted use, distribution, and reproduction in any medium, provided the original work is properly cited.

Stochastic phenomenon has played an important role in various branches of science such as biology, economics, and aircraft. Stochastic modeling approach has achieved a great number of distinguished contributions for a wide spectrum of systems including Markovian jumping systems, Itô stochastic systems, networked control systems with random communication delays, and/or packet losses. Over the past few decades, considerable attention has been paid to modeling, stability analysis, stabilization, robust filtering, model reduction, and practical applications of stochastic dynamical systems. In spite of the extensive and systemic development of stochastic approaches and techniques, there still remain various types of open problems desired to be further strengthened, which includes modeling, filtering, nonparametric methods, system realization and identification, and so forth. Meanwhile, novel and updated developed theories and results are required to be investigated for application of stochastic systems in practical engineering.

This special issue contains thirty-four papers, which are summarized as follows.

"Event-based $H_{\infty}$ filter design for sensor networks with missing measurements" by J. Liu et al. proposes an event triggered mechanism based on sampled-data information, which has some advantages over existing ones. Considering the missing sensor measurements and the network-induced delay in the transmission, a new event-based $H_{\infty}$ filtering is constructed by taking the effect of sensor faults with different failure rates. By using the Lyapunov stability theory and the stochastic analysis theory, sufficient criteria are derived for the existence of a solution to the algorithm of the event-based filter design.

"Finite-horizon robust Kalman filter for uncertain attitude estimation system with star sensor measurement delays" by H.-M. Qian et al. addresses the robust Kalman filtering problem for uncertain attitude estimation system with star sensor measurement delays. Combined with the misalignment errors and scale factor errors of gyros in the process model and the misalignment errors of star sensors in the measurement model, the uncertain attitude estimation model can be established to indicate that uncertainties not only appear in the state and output matrices but also affect the statistic of the process noise. Meanwhile, the phenomenon of star sensor measurement delays is described by introducing Bernoulli random variables with different delay characteristics. A finite-horizon robust Kalman filter is proposed to solve this estimation problem which takes into account the effects of star sensor measurement delays and model uncertainties.

"Robust adaptive fault-tolerant control of stochastic systems with modeling uncertainties and actuator failures" by W. Cai et al. deals with the problem of actuator fault-tolerant control of a class of uncertain stochastic systems with model uncertainties. A robust adaptive control scheme is developed to solve this problem. The proposed approach does not require 
the fault detection and diagnosis roles and thus simplifies the design procedure and achieve a low cost.

"Prescribed performance fuzzy adaptive output-feedback control for nonlinear stochastic systems" by L. Zhang et al. proposes a prescribed performance for a class of singleinput and single-output nonlinear stochastic systems with unmeasured states, and a fuzzy state observer is designed for estimating the unmeasured states. Based on the backstepping recursive design technique and the predefined performance technique, a new fuzzy adaptive output feedback control method is developed. It is shown that all the signals of the resulting closed-loop system are bounded in probability and the tracking error remains an adjustable neighborhood of the origin with the prescribed performance bounds.

"Consensus of multiagent systems with packet losses and communication delays using a novel control protocol" by Z. Yan et al. addresses the consensus problem of multiagent system with packet losses and communication delays under directed communication channels from a practical point of view. A novel control protocol is proposed depending only on periodic sampling and transmitting data in order to be convenient for practical implementation and economical for limited system resources. It is proved that for single integrator agents and double integrator systems with only communication delays consensusability can be ensured through stochastic matrix theory provided that the designed communication topology contains a directed spanning tree. For double integrator agents and high-order integrator agents, the interval system theory is introduced to investigate the consensus of multiagent system.

"State-feedback stabilization for a class of stochastic feedforward nonlinear time-delay systems" by L. Liu et al. investigates the state-feedback stabilization problem for a class of stochastic feedforward nonlinear time-delay systems. By using the homogeneous domination approach and choosing an appropriate Lyapunov-Krasovskii functional, the delayindependent state-feedback controller is explicitly constructed such that the closed-loop system is globally asymptotically stable in probability.

"Robust guaranteed cost observer design for singular Markovian jump time-delay systems with generally incomplete transition probability" by $\mathrm{Y}$. $\mathrm{Li}$ et al. investigates the design of robust guaranteed cost observer for a class of linear singular Markovian jump time-delay systems with generally incomplete transition probability. In this design, each transition rate can be completely unknown or only its estimate value is known. Based on stability theory of stochastic differential equations and linear matrix inequality technique, an observer is designed to ensure that for all uncertainties the resulting augmented system is regular, impulse free, and robust stochastically stable with the proposed guaranteed cost performance.

Of course, the selected issues and papers are not a comprehensive representation of the area of this special issue. Nonetheless, they represent the rich and many-faceted knowledge that we have the pleasure of sharing with the readers.

\section{Acknowledgments}

We would like to express appreciation to the authors for their excellent contributions and patience in assisting us. The hard work of all reviewers on these papers is also very greatly acknowledged.

Ming Liu

Peng Shi

Hamid Reza Karimi

Shen Yin

Xiaojie Su 


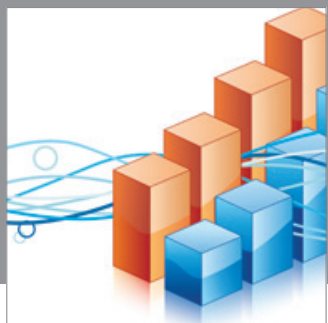

Advances in

Operations Research

mansans

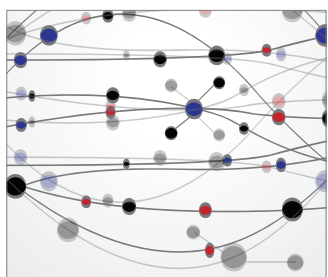

The Scientific World Journal
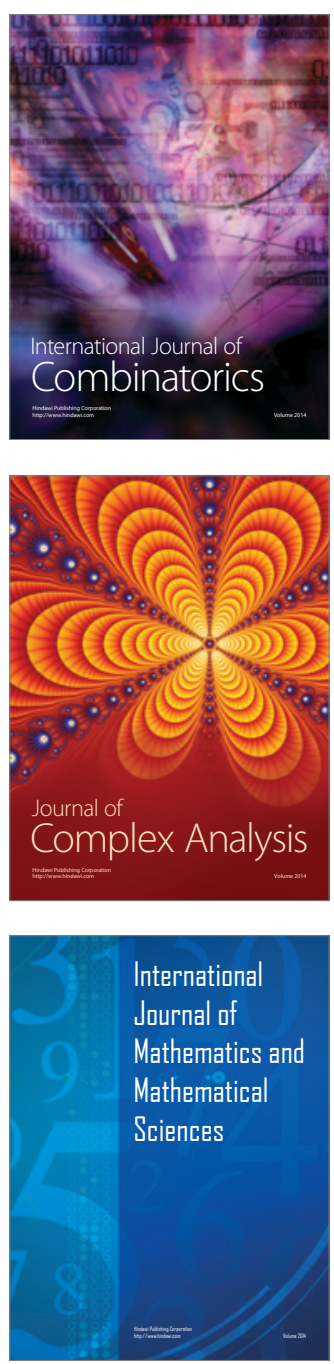
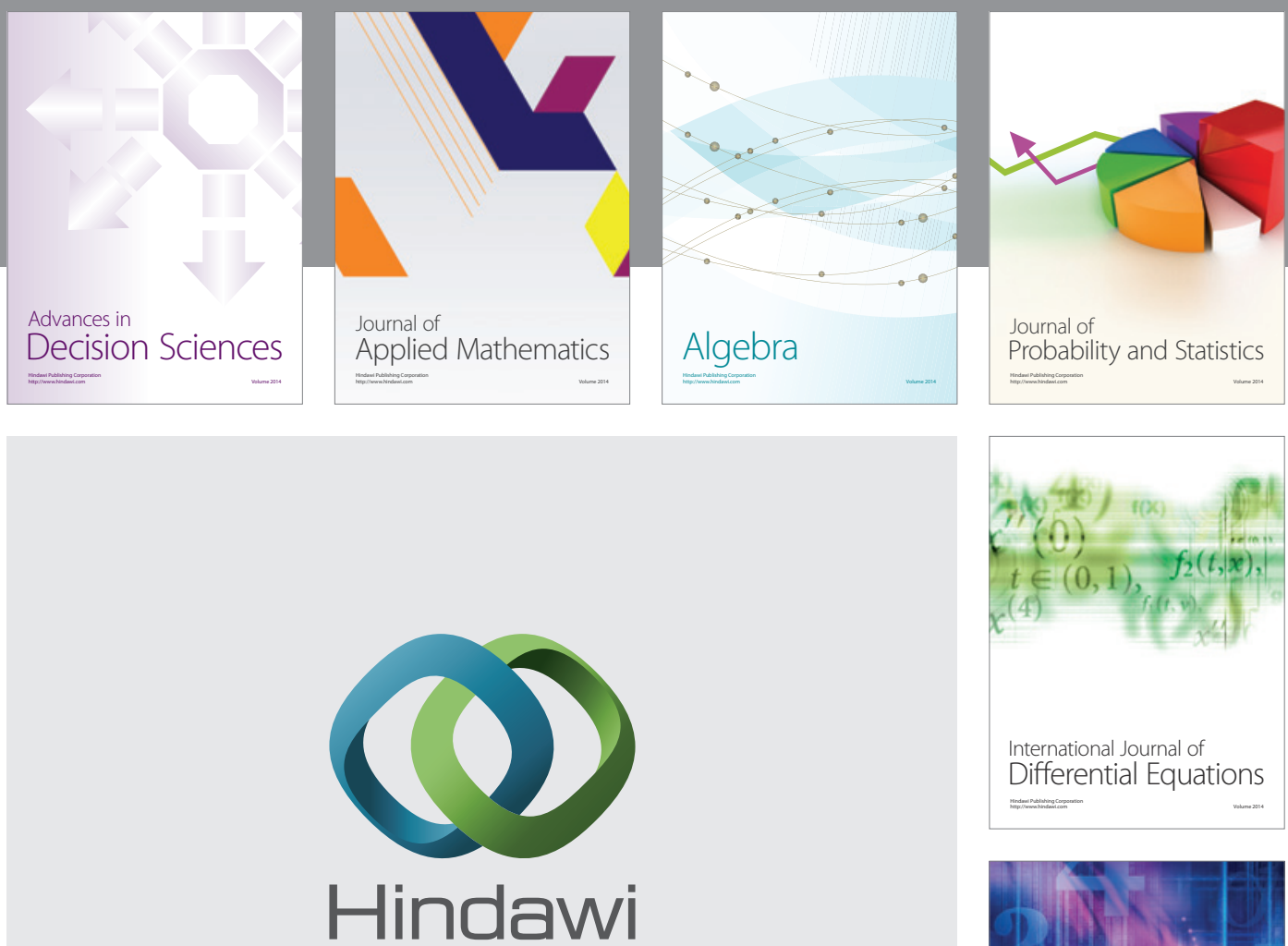

Submit your manuscripts at http://www.hindawi.com
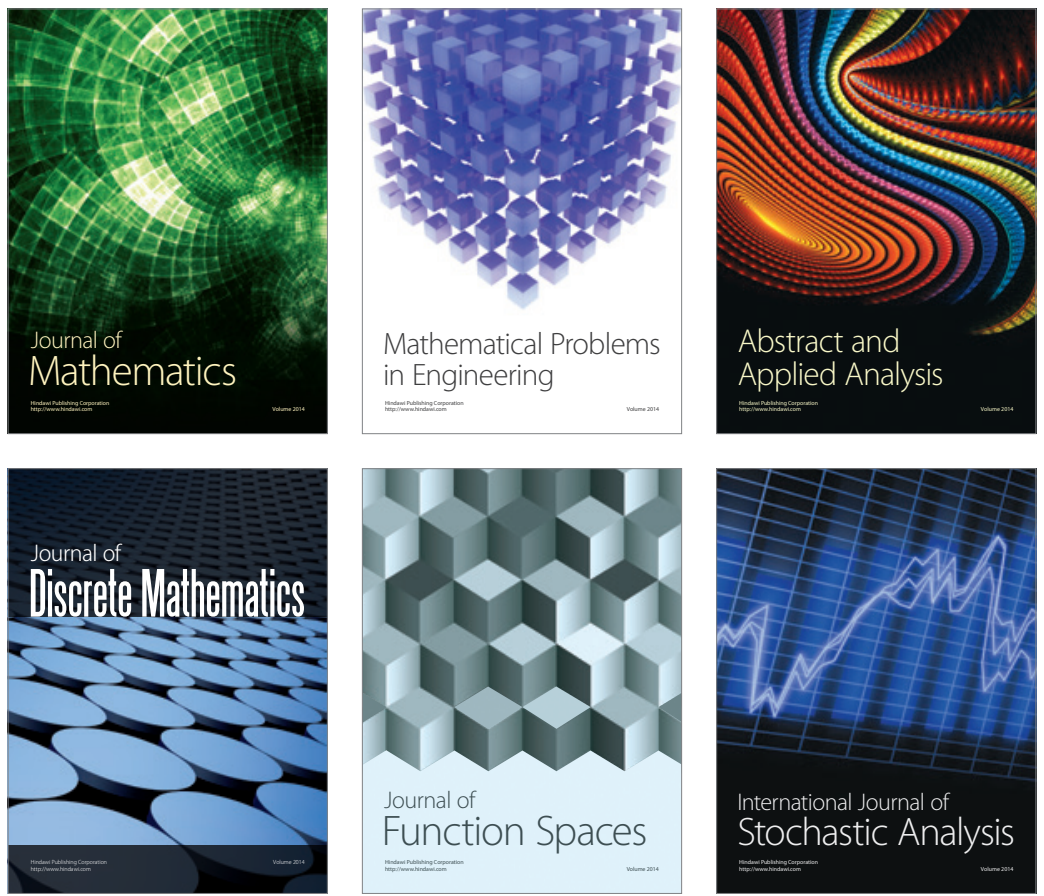

Journal of

Function Spaces

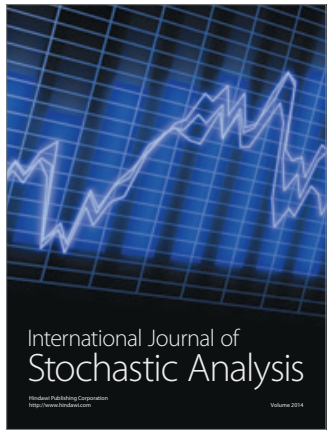

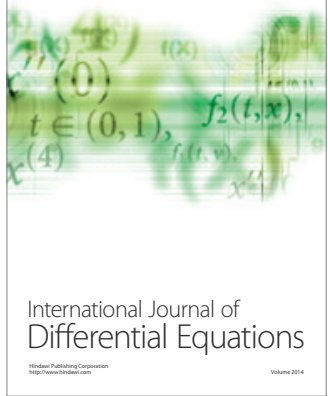
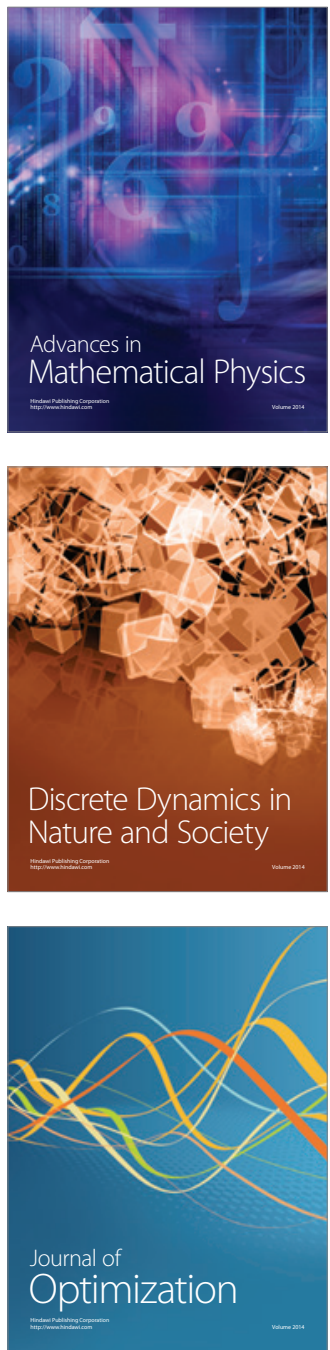